\title{
Structural Design of Agaricus bisporus Picking Robot based on Cartesian Coordinate System
}

\author{
Sun Chenzhe, Hu Xiaomei* , and Yu Tao \\ Shanghai Key Laboratory of Intelligent Manufacturing and \\ Robotics, School of Mechatronic Engineering and \\ Automation, Shanghai University, Shanghai 200072, China \\ *Email: sufeimasohxm@163.com
}

\begin{abstract}
The Agaricus bisporus Picking Robot is one of the researches focuses in the world at present. This paper introduces and analyzes the structure of an Agaricus bisporus Picking Robot based on the independent design. The picking robot complete the picking task by taking the way that the visual area, the picking area and the auxiliary area cooperat2e with each other, the visual-picking work parallelly and taking the working principle of the "three-step walking". Based on the structure principle of Gantry Robot, a rectangular coordinate mechanism with four degrees of freedom is designed. The picking sucker of the robot can complete the three-axis linear motion and the fixed axis rotation in the three-dimensional space. And decompose the space displacement movement into a three-direction linear motion so that the Agaricus bisporus Picking Robot has an advantage of the wide motion space, fast motion and high motion accuracy. In this way, it can quickly complete the fixed-point picking task of Agaricus bisporus Picking Robot. Through the experimental data obtained by the robot picking experiment at the mushroom base, the picking efficiency of the self-developed Agaricus bisporus Picking Robot reached 15s, and the picking success rate reached $91.95 \%$.
\end{abstract}

Keywords-Agaricus bisporus Picking Robot, Gantry Robot, three-step walking

\section{INTRODUCTION}

In recent years, agaricus bisporus is still widely cultivated and consumed in the world, it has a fast growth rate and high yield. However, most of the world's agaricus bisporus production bases still use the labor force to manually pick and classify agaricus bisporus, and some countries produce agaricus bisporus by picking and classifying agaricus bisporus in a semi-automated manner. The manual picking of agaricus bisporus by labor force not only has low picking efficiency, greatly increased labor and increased picking cost, but also cannot be harvested 24 hours a day. Automatic picking of agaricus bisporus will greatly increase the yield of agaricus bisporus [1]2], and it will be an inevitable trend to achieve automatic picking and classification of agaricus bisporus. Based on the existing agricultural picking robot and its working principle [3][4], learning from its mechanical structure, and taking into account the growth environment of agaricus bisporus and the working environment of robot, the agaricus bisporus picking robot was independently developed, and a new type of mushroom picking robot with a four-degree-of-freedom structure based on the rectangular coordinate system to complete the spot picking task was designed[10].

\author{
Lu Xiangyu \\ School of Mechatronic Engineering and Automation \\ University, Shanghai Second Polytechnic University \\ Shanghai, China
}

\section{WORK ENVIRONMENT}

The whole agaricus bisporus cultivation plant maintains constant temperature and humidity, the temperature is controlled at about $18^{\circ} \mathrm{C}$, and the humidity reaches $90 \%$. Therefore, all robot components need the moisture prevention. The growth bed of agaricus bisporus shown in Fig. 1. The Agaricus bisporus Picking Robot needs to work between each layer on the growth bed. Each side of the layer is provided with a circular guide rail with a spacing of $1356 \sim 1360 \mathrm{~mm}$ and a diameter of $20 \mathrm{~mm}$. It is basically flush with the cultivated soil surface and is picked as the moving rail of the robot. The space frame shape of the growth bed is the type of "wide and short", and the internal space has a length of about $20 \mathrm{~m}$, a width of $1400 \mathrm{~mm}$, and a height of about $320 \mathrm{~mm}$. The shape and size of the Agaricus bisporus Picking Robot is mainly limited by the height of the frame space, and the structural design of picking area is most affected. Therefore, due to the work environment and limitation, a structural design scheme developed independently and based on the gantry-type Cartesian coordinate robot was proposed. It's necessary that a safety distance of $4 \mathrm{~mm}$ is left on both sides at the width direction and a safety distance of $30 \mathrm{~mm}$ is left at the height direction to prevent the insecurity caused by the growth bed due to the non-standard processing. So, the width design size is $1392 \mathrm{~mm}$ and the height design size is $290 \mathrm{~mm}$.

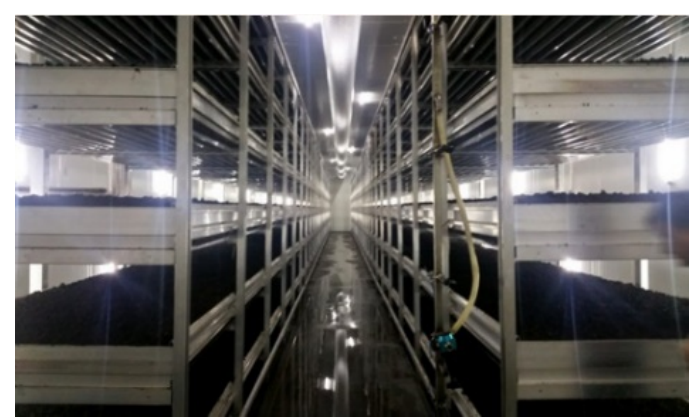

Fig.1 Agaricus bisporus growth bed

\section{WORK PRINCIPLE}

The Agaricus bisporus Picking Robot shown in Fig. 2 is mainly divided into three areas: the visual area, the picking area and the auxiliary area. In order to improve the picking efficiency of the robot, the visual zone is set in front of the picking zone so that the vision-picking parallel working principle is adopted, and agaricus bisporus coordinate points obtained from the vision camera is used as the coordinate 
points of the picking mechanism moving. Under the precondition of the effective shooting range of $200 * 140 \mathrm{~mm}$ and the coordinate points getting precisely in vision area, the whole robot frame needs to move forward by $140 \mathrm{~mm}$ every time to ensure that there is no missing blind spot in the visual area for the calibration of agaricus bisporus. The Agaricus bisporus Picking Robot adopts the basic formula of "threestep walking”: when the visual camera completes the first shot of a row of agaricus bisporus, the robot moves forward by $140 \mathrm{~mm}$; then the robot continues to move forward by the same distance after the second shot of the next row of agaricus bisporus by the visual camera; when the visual camera performs the third shot of agaricus bisporus, the picking mechanism begins to pick the first visually positioned agaricus bisporus, and the robot works repeatedly as described above so that the visual coordinate system and the picking area coordinate system are completely coincident to avoid the appearance of the missing picking of the agaricus bisporus. The visual zone shown in Fig. 3(a) consists of a screw linear module, a vision camera and a light source panel. The screw linear module has high moving precision, and it can meet the demand of $0.02 \mathrm{~mm}$ which achieves the accuracy of the movement error of the coordinate point positioning of the vision system and greatly improves the reliability of the positioning of the vision system. Its installation position is shown in Fig. 3(b), and the visual structure is mounted on the mobile car using 30 aluminum profiles. The picking area consists of a three-axis moving (X, Y, Z axis) rectangular coordinate mechanism and a flat gear rotating mechanism and the agaricus bisporus picking work is completed by a micro vacuum pump, a rotating gear and a suction cup [6]. The auxiliary area is mainly used to install the hardware required for the whole robot, such as electrical cabinet, industrial computer, vacuum pumps, and related connecting components and control components.

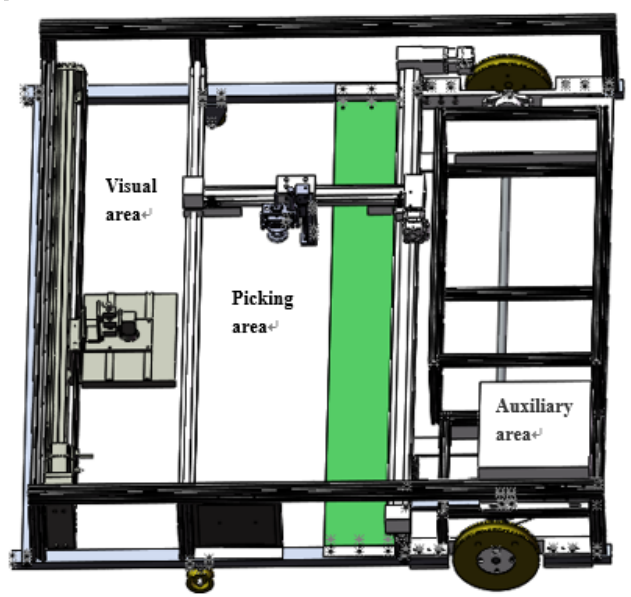

Fig.2 The whole structure of the Agaricus bisporus Picking Robot

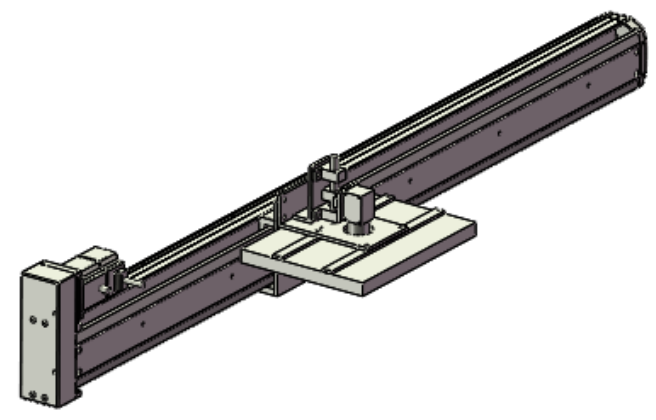

(a)

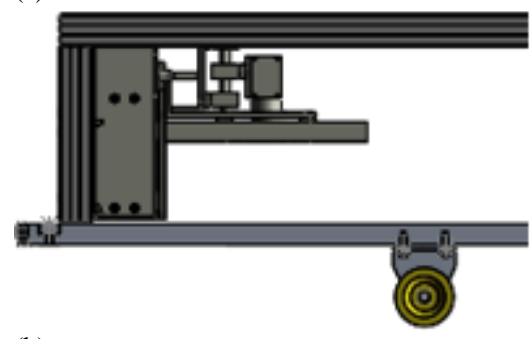

(b)

Fig.3 Structure (a) and instalment (b) of the Vision area

\section{Structural Design Of The Picking Area}

\section{A. Motion unit of Cartesian robot}

When the picking device of the Agaricus bisporus Picking Robot needs to move to a specified position in three dimensions, then the linear motion unit is adopted to satisfy the motion needs [8]. Common linear motion units include: ball screw, ball guide type unit and slide type unit; belt drive, ball guide type unit and slide type unit. The moving distance of the picking device in the $\mathrm{X}$-axis direction is longer, and if the screw type is selected, a large deformation will occur in the case of a large distance, but the belt type can be made long and the deformation effect is small, so X-axis and Yaxis use the toothed belt linear modules that have different effective strokes to achieve their respective movement requirements, the belt drive and slide type unit is suitable for high-speed and high-acceleration movement, and speeding up the moving velocity of the $\mathrm{X}$-axis and $\mathrm{Y}$-axis can improve the picking efficiency of the Agaricus bisporus Picking Robot. While the Z-axis is limited by the space height and the small screw module can meet its motion requirement, therefore, the Z-axis adopts a small screw straight linear module.

\section{B. Structural design based on Cartesian coordinate system}

On the basis of determining the linear unit of the Agaricus bisporus Picking Robot based on the Cartesian coordinate system [7][8] and the structural principle of the Gantry type Cartesian robot [9], the mechanical structure design of the picking area of the Agaricus bisporus Picking Robot was carried out. The basic structure is shown in Fig.4: the moving distance in the $\mathrm{X}$-axis and $\mathrm{Y}$-axis directions is longer, and the synchronous belt linear module is used. One end of the Y-axis module is lapped and fixed on the sliding of the $\mathrm{X}$-axis linear module, and the other end is lapped and fixed on a sliding guide parallel to the $\mathrm{X}$-axis. The slider on the $\mathrm{X}$-axis module is moved at the direction driven by the steeper motor with the encoder; the Y-axis module follows the $\mathrm{X}$-axis slider to finish the moving on the one hand, and the movement in the $\mathrm{Y}$-axis direction is driven by the selfcontained stepper motor with the encoder on the other hand. the space height at the $\mathrm{Z}$-axis direction is small, so the smaller-sized screw linear module is used with the microcylinder for segmentation extend and shrink. In the way, the effective extend and shrink in the Z-axis direction is completed by the supporting motor and the vacuum pump. The choice of micro-cylinders allows for a large up and down telescopic distance in an efficient space to meet the corresponding picking and collection size requirements. The picker installation location is shown in Fig. 5, which framed on the mobile car using the 30 profiles. 


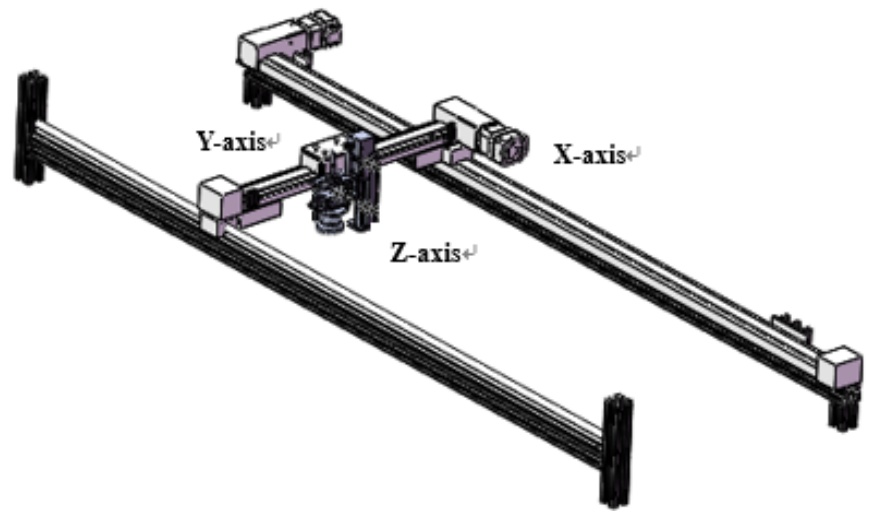

Fig.4 Basic structure of the picker

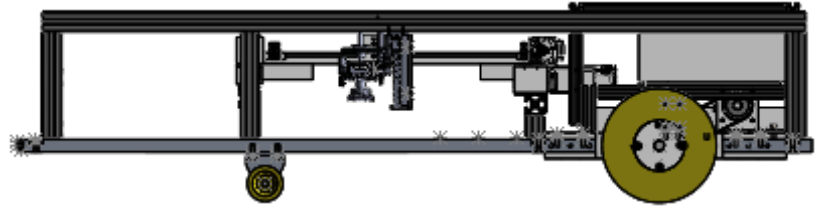

Fig.5 Instalment location of the picker

\section{BASE EXPERIMENT}

After a period of R\&D design and machining assemblage, the first-generation prototype of the Agaricus bisporus Picking Robot was successfully finished. After the joint debugging of vision system, control system and mechanical devices in the laboratory simulation of the culture environment of Agaricus bisporus, the simulation experiment of the visual recognition and extraction of the coordinates of agaricus bisporus and the automatic spotting of the picking device under the control system has a good effect. The threeaxis moving speed of the Agaricus bisporus Picking Robot based on the Cartesian coordinate system is fast and the moving velocity is adjustable. Under the precondition of good picking effect in the simulated environment, the robot was tested in the real environment of agaricus bisporus base, and experimental data recorded were summarized and analyzed, which provided the direction for the improvement and optimization of the Agaricus bisporus Picking Robot.

\section{A. Identification and picking data records}

The Agaricus bisporus Picking Robot was placed on the growth bed, and the single experimental area was $420 * 1000 \mathrm{~mm}$. In this experiment, the organ sponge suction cup with a diameter of $40 \mathrm{~mm}$ was used and the vacuum degree that is $-35.4 \mathrm{KPa}$ was adopted. The following shown in Table I is the experimental data for multiple picking.

TABLE I. EXPERIMENTAL DATA

\begin{tabular}{|c|c|c|c|c|c|}
\hline $\begin{array}{c}\text { Group } \\
\text { num }\end{array}$ & $\begin{array}{c}\text { Area } \\
(\mathbf{m m} \boldsymbol{*} \boldsymbol{m m})\end{array}$ & Total & Visual & Actual & Miss \\
\hline 1 & $420 * 1000$ & 45 & 33 & 30 & 3 \\
\hline 2 & $420 * 1000$ & 50 & 49 & 42 & 7 \\
\hline 3 & $420 * 1000$ & 40 & 38 & 34 & 4 \\
\hline 4 & $420 * 1000$ & 50 & 46 & 41 & 5 \\
\hline 5 & $420 * 1000$ & 55 & 52 & 48 & 4 \\
\hline 6 & $420 * 1000$ & 40 & 37 & 31 & 5 \\
\hline 7 & $420 * 1000$ & 43 & 40 & 37 & 3 \\
\hline 8 & $420 * 1000$ & 50 & 46 & 41 & 5 \\
\hline 9 & $420 * 1000$ & 55 & 53 & 49 & 4 \\
\hline 10 & $1400 * 1000$ & 143 & 132 & 113 & 19 \\
\hline
\end{tabular}

\section{B. Data analysis}

From the visual identification and the actual picking data it can be figured that there is a larger fluctuation in success rate from the identification of position to the completion of picking, and the reasons may be as follows.

- From the actual picking process at the scene, it can be found that most of the agaricus bisporus was not harvested because the stroke in the Z-axis direction was not reached shown in Fig. 6 or the size of the agaricus bisporus was small, or the growth tilt shown in Fig. 7 caused the gas leakage at the suction cup when the picking did not reach the corresponding suction pressure.

- Due to the displacement error of the robot frame moving forward and backward, the proposed visual positioning point coordinate system deviates from the picking area positioning point coordinate system, and the picking device still moves in the light of the original coordinate point, and there is a bias with the actual agaricus bisporus center point so that it is not picked successfully.

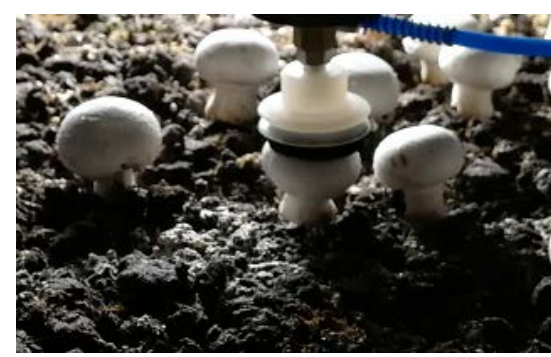

Fig.6 Insufficient Z-axis travel

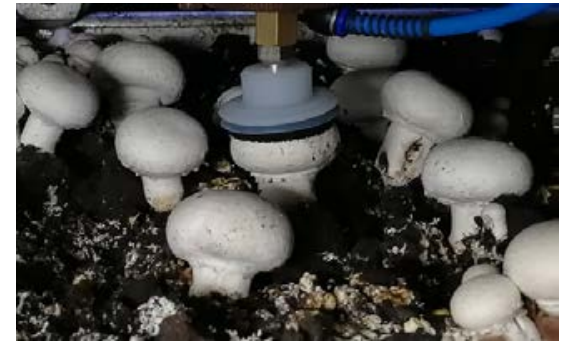

Fig.7 Growth tilt of agaricus bisporus

\section{Analysis of picking results}

According to the experimental data of the base and the situation of the scene, although the robot has fluctuated in the picking success rate, it still reached 91.95\%. The visual recognition and localization rate of the Agaricus bisporus Picking Robot to the agaricus bisporus reached 93.56\%, and the picking speed reached 15s. Based on the rectangular coordinate system, the Agaricus bisporus Picking Robot still needs to be improved and optimized in many aspects. From the picking data, it is analyzed that the picking robot still needs to improve the Z-axis stroke and the frame movement error, but the three-axis structure of the rectangular coordinates of the picking area still achieves a good rapid movement and picking effect.

\section{CONCLUSION}

According to the kind of Agarics bisporus Picking Robot that was developed independently is capable of automatic 
picking. Under the premise of limited space, the working principle of "three-step walking" and the way of visualpicking parallel work were used in the robot. Based on above, the corresponding mechanical structure design was carried out. The picking area draws on the gantry-type rectangular coordinate structure principle, and adopted a kind of picking structure based on the Cartesian coordinate system. Base experiment data shows that the Agarics bisporus Picking Robot based on the Cartesian coordinate system has higher working precision and stability, and the robot has better working efficiency and picking success rate, which lays a solid foundation and accumulates valuable experience for the improvement and optimization of the Agarics bisporus Picking Robot.

\section{REFERENCES}

[1] Yue Jianrong, Guo Hui, Zhang Xuejun, Yang Wanzhang, Zhou Yansheng. Discussion on the application of picking robot in agriculture[J].Xinjiang Agricultural Mechanization, 2016(01):31-34.

[2] LZhang Junxiong, He Fen. Research Progress in Facility Agricultural Picking Robots[J]. Contemporary Agricultural Machinery, 2016(1): 22-24.

[3] LI Binghe, URA Takanori, FUJINAGA Takuya, YASUKAWA
Shinsuke, SONODA Takashi, ISHII Kazuo. Development of EndEffector for Harvesting Tomato Using Suction and Cutting Mechanism[J]. The Proceedings of JSME annual Conference on Robotics and Mechatronics (Robomec),2017,2017(0).

[4] NAKAO Kosuke,MIKI Haruka,NAKAMURA Gaichi, HAMAMATSU Hiroshi, MATSUO Takayuki. Development of movement mechanism of tomato harvesting robot for two kinds of environments[J]. The Proceedings of JSME annual Conference on Robotics and Mechatronics (Robomec), 2017, 2017(0).

[5] Liu Pengli. Design and research of apple picking robot [D]. Xi'an University of Technology, 2018

[6] Liu Jizhan, Li Pingping, Ni Qi, et al. Design and Experiment of Vacuum Pickup Device for Tomato Picking Robot[J]. Transactions of the Chinese Society of Agricultural Machinery, 2010, 41(10): 170173.

[7] Ge Xinsheng, Liu Song, Zhang Yong. Complete Cartesian Coordinate Method for Robot Dynamics Analysis[J]. Mechanical Design, 2001(10): 13-15+30-0.

[8] Zhao Yuxin. Research on Cartesian robot system based on linear motion unit[D].Qilu University of Technology, 2014

[9] Zhang Jun, Zhu Haitao, Tan Dingzhong. Research on Cross Gantry Robot[J]. Mechanical Engineer, 2007(01): 116-118.

[10] Lu Junyi, Yang Qinghua, Gao Feng, et al. Trajectory Planning of Five Degrees of Freedom Agricultural Picking Robot[J]. Mechanical Engineering, 2010, 27(12):1-6. 